\title{
VALIDEZ DE UNA ESCALA DE GESTIÓN DEL CONOCIMIENTO EN INSTITUCIONES DE EDUCACIÓN BÁSICA
}

\section{SCALE VALIDATION OF A KNOWLEDGE MANAGEMENT IN THE ELEMENTARY EDUCATION INSTITUTIONS}

\section{Claudia Patricia Salgado Ibarra}

Profesora-Investigadora de la Universidad de Guanajuato (México).

E-mail: csalgado@ugto.mx

\section{Martha Ríos-Manríquez}

Profesora-Investigadora de la Universidad de Guanajuato (México)

E-mail: martha@ugto.mx

\section{María Dolores Sánchez-Fernández}

Profesor e Investigador de la Universidad de La Coruña (España).

E-mail: msanchezf@udc.es 


\title{
VALIDEZ DE UNA ESCALA DE GESTIÓN DEL CONOCIMIENTO EN INSTITUCIONES DE EDUCACIÓN BÁSICA
}

\section{RESUMEN}

El presente artículo tiene por objetivo obtener un instrumento robusto que evalúe la Gestión del conocimiento en Instituciones de Educación Básica en el Estado de Guanajuato, México, a partir de la adaptación de los modelos de diagnóstico "Knowledge Management Assessment Tool" (KMAT) y el "Centro de Sistemas de Conocimiento" (CSC). El primer modelo contempla cuatro dimensiones: liderazgo, medición, cultura y tecnología y el segundo modelo considera seis factores: entendimiento, intención, requerimientos, práctica, resultados y visión Futura. Para lograr este objetivo, se utilizó un análisis factorial exploratorio, con factores de extracción de componentes principales, y con el método Varimax de rotación ortogonal al instrumento de 47 ítems, sobre una muestra de 222 docentes y directivos de 40 escuelas de educación básica. Los resultados indujeron a la eliminación de 8 ítems que modifican el modelo propuesto por CSC, eliminando el factor de intención y favoreciendo los factores práctica y visión futura. En relación a las variables KMAT, se incrementó su nivel de confiabilidad en cada una, aunque el factor medición obtuvo un valor de confiabilidad bajo, pero suficiente para considerar como resultado final una escala robusta.

Palabras clave: gestión del conocimiento; Instituciones de Educación Básica; análisis factorial; México.

\section{SCALE VALIDATION OF A KNOWLEDGE MANAGEMENT IN THE ELEMENTARY EDUCATION INSTITUTIONS}

\begin{abstract}
The present paper establishes a new robust tool that evaluates the Knowledge Management at the Elementary Education Institutions in Guanajuato, Mexico, this new model is based on two diagnostic models; the first is "Knowledge Management Assesment Tool" (KMAT) which contemplates the variables leadership, measurement, culture and technology and the second method is the "Center of Knowledge systems" (CKS) which includes six variables: understanding, intention, requirements, practice, results and further vision. The new tool was obtained by an exploratory factor analysis, that used extraction factors of principal components and a Varimax method of orthogonal rotation, in a test of 47 items where 222 professors and directors from 40 schools were interviewed. The results showed that 8 items needed to be eliminated since they modified the proposed model by the CKS, for example the intention factor was no needed while practice and further vision seemed to favor the model. Otherwise, all of the KMAT variables confidence increased, even if the measurement factor was obtained with less confidence but with the enough to be considered into the robust scale.
\end{abstract}

Revista de Gestão e Secretariado-GeSec, São Paulo, v. 8, n. 2, p 20-39, Mai./Ago. 2017. 
Keywords: knowledge management; basic education institutions; leadership; Exploratory Factor Analysis, Mexico.

\section{INTRODUCCIÓN}

La gestión del conocimiento como disciplina ha tenido un desarrollo fundamental dentro de las empresas y las organizaciones, la Organización para la Cooperación y el Desarrollo Económico (OCDE, 1996) reconoce el conocimiento como el motor de la productividad y del crecimiento económico, así como la necesidad de entender y medir las actividades de gestión de conocimiento, para que las organizaciones y sus sistemas puedan mejorar lo que hacen y para que las administraciones puedan desarrollar políticas públicas que promuevan estos beneficios (OCDE, 2003), para ello requieren de gestionar el conocimiento en sus organizaciones, siendo las instituciones educativas las que apoyan tanto en el entorno público o privado (Gianini y Gerardin, 2010).

En el ámbito de las instituciones escolares la gestión del conocimiento es un fenómeno del que se está tomando conciencia en forma reciente y del que aún no se puede hablar como un campo de conocimiento y práctica ya constituido (Minakata, 2009). Situación que se ve reflejada en el sistema de educación básica de México donde actualmente, no se encontraron en la literatura estudios publicados que nos puedan indicar el estado de la gestión del conocimiento en su interior, lo que nos lleva a investigar en este reporte un instrumento adecuado a la idiosincrasia mexicana, que permita evaluar la situación actual de la gestión del conocimiento de las Instituciones de educación Básica del Estado de Guanajuato.

Así, el artículo se organiza de la siguiente manera: en la segunda sección se presenta un panorama general de la educación básica en el mundo y en México, así como un resumen del estado de la gestión del conocimiento y su vinculación al sector educativo mediante los elementos rectores bases de la presente investigación y del instrumento diagnóstico, de donde se deducen las hipótesis. La tercera sección describe la metodología utilizada. En la cuarta sección se analizan y discuten los resultados. La última sección se presentan las consideraciones finales.

\section{Revisión de la Literatura}

En este apartado se desarrollan los temas ejes de esta investigación que son la educación básica, conocimiento en la educación, gestión del conocimiento, modelos de gestión de conocimiento en el sector educativo, modelo KMAT y CSC.

\subsection{Educación Básica}

La educación básica primaria y secundaria de acuerdo con la Clasificación Internacional Normalizada de Educación (CINE) de las Naciones Unidas es aquella que se enfoca principalmente a proporcionar a los estudiantes destrezas básicas en lectura, escritura y matemáticas y sentar una sólida base para el aprendizaje y la comprensión de las áreas esenciales del conocimiento, así como establecer las bases para el desarrollo humano y el

Revista de Gestão e Secretariado-GeSec, São Paulo, v. 8, n. 2, p 20-39, Mai./Ago. 2017. 

aprendizaje a lo largo de la vida (CINE 2011, 2013). Es también ampliamente reconocida como un derecho humano fundamental y un instrumento clave para mitigar la pobreza y el desarrollo humano sostenible. En la mayoría de los países, el Estado asume una función preponderante en la gestión y el financiamiento del sistema educativo, especialmente a nivel de educación primaria (UNESCO, 2007).

En Iberoamérica, el acceso a la educación primaria es homogéneamente alto, salvo excepciones, alcanzando niveles cercanos a la universalidad en varios países. Las tasas de conclusión de la enseñanza primaria son de $96 \%$ en las zonas urbanas y solo de $85 \%$ en las rurales (OEI, 2010). Entre el $40 \%$ y el $60 \%$ de los alumnos latinoamericanos participantes en el Programa para la Evaluación Internacional de Estudiantes (PISA) no alcanza los niveles de rendimiento que se consideran imprescindibles para incorporarse a la vida académica, social y laboral como ciudadano (OEI, 2010). Al menos uno de cinco estudiantes de 15 años de edad de los países que integran la Organización para la Cooperación y Desarrollo Económico (OCDE) no alcanza el nivel mínimo de habilidades que demanda la sociedad actual, existiendo una gran diferencia entre los estudiantes de los diferentes bloques socioeconómicos (OCDE, 2015). Los países que logran mejores resultados en las evaluaciones internacionales cuidan especialmente a su profesorado: seleccionan a los candidatos a la formación docente; ofrecen buenos salarios iniciales y presentan múltiples oportunidades de mejora durante la carrera profesional (Ravela, 2000). En este nivel, los modelos de educación basados en competencias son una tendencia educativa importante a nivel internacional, principalmente para la Comunidad Europea, anglosajona, para América Latina y México (Díaz Barriga y Lugo, 2003).

En México por su carácter de obligatoriedad, el número de población y por ende el número de instituciones a nivel básico, la educación básica es el más importante de los subsistemas que comprende servicios educativos en los niveles de educación preescolar, primaria y secundaria. Su baja calidad de la enseñanza y niveles de logro insatisfactorios en comparación con los niveles de logro de estudiantes de otros países con niveles de desarrollo similares son determinados por las evaluaciones de PISA, en el 2012 el 55\% de los alumnos mexicanos no alcanzó el nivel de competencias básicas en matemáticas y en lectura el $41 \%$ de los alumnos no alcanza el nivel de competencias básico (OCDE, 2012). No obstante, el alto nivel de gasto corriente, con limitado margen para la inversión que en 2010, México ocupaba el primer lugar entre los países de la OCDE en gasto público asignado a la educación como proporción del gasto público total, que fue de $20.5 \%$ en comparación con el $12.4 \%$ gasto promedio de los países que integran la OCDE (OCDE, 2013). Este pobre desempeño de la educación básica en México en buena medida se debe a la compleja composición y carácter disfuncional del sistema educativo federal, con una verticalidad en la toma de decisiones, subordinado al poder político y al papel del sindicato o coordinadora nacional de trabajadores de la educación y su relación con el poder político en México (Muñoz, 2008).

2.2 El conocimiento en la educación

La educación representa para el ser humano una construcción continua de sus conocimiento y aptitudes y de su facultad de juicio y acción, además de ocupar un lugar cada vez mayor en la vida de los individuos a medida que aumenta su función en la dinámica de las sociedades modernas (Delors, 1996). La apropiación del conocimiento se da por medio del aprendizaje, el aprendizaje y desarrollo de competencias ha sido un proceso complejo que ha generado numerosas interpretaciones y teorías de cómo se efectúa realmente. Cuatro de las diferentes teorías existentes de este proceso son el conductismo, el cognitivismo, el constructivismo y el conectivismo. La distinción básica entre estas cuatro teorías radica en la

Revista de Gestão e Secretariado-GeSec, São Paulo, v. 8, n. 2, p 20-39, Mai./Ago. 2017. 
forma en que conciben el conocimiento. Para el conductismo, el conocimiento consiste fundamentalmente en una respuesta pasiva y automática a factores o estímulos externos que se encuentran en el ambiente (Marí y Onrubia, 2002). El cognitivismo considera el conocimiento básicamente como representaciones simbólicas en la mente de los individuos (Snelbecker, 1983). El constructivismo, concibe el conocimiento como algo que se construye, que se elabora a través de un proceso de aprendizaje, es una elaboración individual relativa y cambiante (Bednar, Cunningham, Duffy y Perry, 1992). En el constructivismo social, el conocimiento es un proceso de interacción entre el sujeto y el medio social y cultural (Vigotsky, 1978), está teoría enfatiz la idea de que el conocimiento no solo se construye de forma individual en la mente del sujeto sino que hay una construcción dinámica y cambiante de origen social y cultural, la persona aprende a través de la internalización del conocimiento socialmente construido (Ayuste, Gros y Valdivieso, 2012). En el conectivismo Downes (2007), define el conocimiento conectivo como el conocimiento resultante entre dos entidades conectadas, donde una de las propiedades de una de ellas debe conducir a, o convertirse en, propiedad de la otra entidad. La conectividad permite que los individuos creen y distribuyan sus propios materiales e identidad (Simens, 2004).

La sociedad del conocimiento es una sociedad del aprendizaje (Hargreaves, 2003), donde el conocimiento es el principal elemento del modelo emergente de producción, y el aprendizaje es su proceso más importante para lograrlo (OCDE, 2000). En la sociedad del conocimiento, la educación se concibe como un proceso integral, donde el conocimiento adquirido a través de ella, está pasando a ser el activo intangible más importante del paradigma productivo de la sociedad del siglo XXI (Aguerrondo, 1999), donde la educación ejercerá un rol estratégico en la potencialización del conocimiento del ser humano y el desarrollo sustentable de la sociedad (DOF, 2013).

Entre los principales retos que enfrenta el sector educativo en la sociedad del conocimiento son: brindar una educación que contemple conocimientos y aprendizajes enfocados mayoritariamente a la adquisición de contenidos de alto orden, es decir, contenidos de mayor utilidad y complejidad y el desarrollo de competencias básicas para la vida (Hernández y Velasco, 1990), donde la educación tradicional coexista con la educación virtual y la educación corporativa de las propias empresas y dar nuevas respuestas en lo que algunos autores han llamado la "Sociedad del Aprendizaje del Conocimiento Social" (Dalhamn, Carl y Aubert, 2001). Es este contexto, la escuela sigue desempeñando una función crucial en la sociedad del conocimiento como institución fundamental para la formación integral de los ciudadanos, y un proyecto que persigue la eficacia y la vinculación de la educación con las necesidades de desarrollo del país (SEP, 2016).

\subsection{Gestión del conocimiento}

La Gestión del conocimiento es una disciplina emergente que tiene por objetivo generar, compartir y utilizar el conocimiento existente en un determinado espacio, para dar respuesta a las necesidades de los individuos y de las comunidades en su desarrollo (Peluffo y Catalán, 2002). Para Davenport y Prusak (2000), el conocimiento es una mezcla de experiencia, valores, informaci n y "saber hacer" que sirve como marco para la incorporaci n de nuevas experiencias e información, y es útil para la acción. Se origina y aplica en la mente de los conocedores. Nonaka y Takeuchi (1995), en su Modelo Proceso para la Creación del Conocimiento distinguen la dimensión epistemológica del conocimiento en conocimiento 
tácito y explícito. Taiwana (2002), define el conocimiento tácito como aquel que forma parte de las experiencias de aprendizaje personales de cada individuo, es personal, propio del contexto y, por tanto, difícil de formalizar y comunicar, abarca creencias, imágenes, intuiciones, modelos mentales y habilidades técnicas, valores y perspectiva. Este conocimiento puede ser transferido a través del entrenamiento o ser adquirido de la experiencia personal (Alwis y Hartmann, 2008). El conocimiento explícito o codificado lo define Taiwana (2002), como aquel que puede ser estructurado, almacenado y distribuido por lo que puede describirse, documentarse y comunicarse entre las personas, a través de un lenguaje formal y sistemático de forma directa en forma de textos, videos, sonido, software, entre otros.

En la medida en que el conocimiento intelectual se agrupa y se comparte en una organización, se convierte en un cimiento sólido para el desarrollo de su ventaja competitiva. Davenport y Prusak (2000) y Hawryszkiewycz (2009), consideran que en las organizaciones, el conocimiento se deriva de la información, obtenida de los datos, la información asociada a un contexto y a una experiencia se convierte en conocimiento, el conocimiento asociado a una organización y a una serie de capacidades organizativas se convierte en capital intelectual. Steward (1997), define el capital intelectual como material intelectual, conocimiento, información, propiedad intelectual, experiencia, que puede utilizarse para crear valor. Es fuerza cerebral colectiva siendo difícil de identificar y aún más de distribuir eficazmente. Pero quien lo encuentra y lo explota, triunfa.

Mientras que la gestión del conocimiento (GC) es descrita como la práctica de aprovechar el conocimiento como ventaja competitiva (Nissen (2006). En la educación la GC es el proceso que continuamente asegura el desarrollo y aplicación de todo tipo de conocimientos de valor en un centro educativo para mejorar su rendimiento y resultados, así como sus ventajas competitivas (Canton, 2009). Es considerado también como una herramienta que proporciona elementos claves a los directores y al personal de las instituciones educativas para enfrentar el reto de la era del conocimiento, de conocer el beneficio de crear y compartir el conocimiento y como medio de mejora del proceso de enseñanza aprendizaje (Sallis y Jones, 2002).

Existen diversos factores que influyen en la gestión del conocimiento, en el ámbito educativo Stankosky (2005), de la universidad de Washington categoriza cuatro factores clave para la gestión del conocimiento: liderazgo, organización, aprendizaje y tecnología. Chungchi and Chein-Chung (2013), consideran la tecnología de la información, la cultura organizacional, la estructura orgánica, la organización de aprendizaje, el liderazgo, el sistema de recompensas, y la competencia del personal como factores clave en una escuela primaria de Taiwán.

\subsection{Modelo de Gestión del conocimiento en el sector educativo}

En la literatura se encontraron diferentes modelos de gestión del conocimiento en la educación: Modelo Sallis y Jones. Gestión del conocimiento en la educación (Sallis y Jones, 2002); Modelo de los diez pasos del mapa del conocimiento (Taiwana, 2002); Modelo Intelect y de su evolución en el Modelo Intellectus para dirigir y gestionar el conocimiento en las universidades (CIC-IADE, 2003); Modelo Cranfield y Taylor (Cranfield y Taylor, 2008); Modelo en laboratorios académicos (Jaime, Gardoni, Mosca y Vinck, 2005); Modelo Accelera de creación y gestión del conocimiento (Gairín, Rodríguez-Gómez, 2012); Modelo

Revista de Gestão e Secretariado-GeSec, São Paulo, v. 8, n. 2, p 20-39, Mai./Ago. 2017. 
de gestión del conocimiento del Centro de Investigación de Excelencia (CIE) de Colombia (Castro 2011, García, Camarena y Gil, 2009, y Dutta y De Meyer, 1997); Modelo institucional de gestión del conocimiento. Universidad CES (Lopera y Quiroz, 2013); Modelo de gestión del conocimiento en educación primaria (Chungchi y Chien-Chung , 2013); y el Modelo Knowledge Management Assessment Tool, desarrollado por la American Productivity y Quality Center (APQC, 2015) y Andersen (1999).

\subsection{Modelo KMAT y CSC}

Del análisis de los diferentes modelos, para esta investigación se eligio el modelo Knowledge Management Assessment Tool (KMAT) (APQC, 2015; Andersen, 1999), porque permite evaluar el nivel de conocimiento en una organización a partir de un instrumento de evaluación y diagnóstico fundamentado en seis elementos del proceso de gestión del conocimiento: la creación, identificación, colección, adaptación, aplicación y difusión del conocimiento, integrados en cuatro factores facilitadores: liderazgo, cultura, medición y tecnología. Factores evaluados en las dimensiones de entendimiento, intención, requerimientos práctica, resultados y visión futura por el Consorcio para la Administración del Conocimiento del Centro de Sistemas de Conocimiento (CSC, 2001), véase tabla 1.

Tabla 1. Factores del Modelo KMAT y CSC

\begin{tabular}{|c|c|c|c|}
\hline Modelo KMAT & Descripción & $\begin{array}{c}\text { Factores de } \\
\text { evaluación del } \\
\text { CSC }\end{array}$ & Descripción \\
\hline Liderazgo & $\begin{array}{l}\text { Establece la estrategia y la misión } \\
\text { de las organizaciones y como éstas } \\
\text { utilizan el conocimiento para } \\
\text { mejorar sus competencias } \\
\text { distintivas }\end{array}$ & Entendimiento & $\begin{array}{l}\text { Es el grado en que el ejecutivo } \\
\text { percibe estar familiarizado } \\
\text { con la gestión del } \\
\text { conocimiento }\end{array}$ \\
\hline \multirow{2}{*}{$\begin{array}{l}\text { Cultura } \\
\text { organizacional }\end{array}$} & \multirow{2}{*}{$\begin{array}{l}\text { Son los mecanismos y acciones } \\
\text { que favorecen el aprendizaje y la } \\
\text { innovación incluyendo aquellas } \\
\text { acciones que refuerzan el } \\
\text { comportamiento abierto al cambio } \\
\text { y al nuevo conocimiento en la } \\
\text { organización }\end{array}$} & Intención & $\begin{array}{l}\text { Detección la orientación } \\
\text { existente, en el ejecutivo y de } \\
\text { la organización en que trabaja } \\
\text { haca el uso de la gestión del } \\
\text { conocimiento }\end{array}$ \\
\hline & & Requerimientos & $\begin{array}{l}\text { Los elementos de soporte } \\
\text { percibidos por ele ejecutivo } \\
\text { como necesarios para iniciar } \\
\text { en su organización proceso de } \\
\text { gestión del conocimiento }\end{array}$ \\
\hline \multirow[t]{2}{*}{$\begin{array}{l}\text { Tecnología } \\
\text { información y la } \\
\text { comunicación }\end{array}$} & \multirow{2}{*}{$\begin{array}{l}\text { Sistemas técnicos y ordenados que } \\
\text { la organización utiliza para } \\
\text { recoger, almacenar y diseminar el } \\
\text { conocimiento como apoyo a la } \\
\text { gestión del conocimiento en la } \\
\text { organización }\end{array}$} & Práctica & $\begin{array}{l}\text { Conocer los procesos y } \\
\text { sistemas de gestión del } \\
\text { cocimiento que están siendo } \\
\text { implantadas en las } \\
\text { organizaciones }\end{array}$ \\
\hline & & Resultados & $\begin{array}{l}\text { Valor agregado por la gestión } \\
\text { del conocimiento a las } \\
\text { organizaciones que lo están } \\
\text { practicando }\end{array}$ \\
\hline
\end{tabular}

Revista de Gestão e Secretariado-GeSec, São Paulo, v. 8, n. 2, p 20-39, Mai./Ago. 2017. 


\begin{tabular}{|l|l|l|l|}
\hline Medición & $\begin{array}{l}\text { Establece los indicadores del } \\
\text { capital intelectual y la distribución } \\
\text { de los recursos para potenciar el } \\
\text { conocimiento que mejore la } \\
\text { competitividad de la organización }\end{array}$ & Visión Futura & $\begin{array}{l}\text { Pronóstico que los ejecutivos } \\
\text { tienen sobre la permeabilidad } \\
\text { de la gestión del conocimiento } \\
\text { a las empresas }\end{array}$ \\
\hline
\end{tabular}

Fuente: Elaboración propia a partir del modelo KMAT (APQC, 2015) Andersen (1999) y la evaluación de factores del CSC (2001).

\subsection{Análisis factorial Exploratorio}

El Análisis factorial exploratorio permite identificar una distribución de factores (Pérez, Medrano, 2010), reduciendo el número de indicadores, donde las variables sean explicadas por un número más reducido de factores (Kline (2000), a fin de que estos expliquen significativamente la varianza total del instrumento estudiado.

\section{Metodología}

Con el objetivo de obtener un instrumento robusto que evaluá la Gestión del conocimiento en Instituciones de Educación Básica en el Estado de Guanajuato, México, a partir de la adaptación del modelo de diagn stico "Knowledge Management Assessment Tool", que contempla cuatro dimensiones: liderazgo, medición, cultura y tecnología. Y de la evaluación propuesta del Consorcio para la Administración del Conocimiento del Centro de Sistemas de Conocimiento (CSC, 2001), considerando seis factores: entendimiento, intención, requerimientos, práctica, resultados y visión futura, se realizó un Análisis Factorial Exploratorio, con factores de extracción de componentes principales, utilizando el método Varimax de rotación ortogonal.

\subsection{Instrumento}

El instrumento fue modificado a partir de las características propias de las instituciones de educación básica en base a la evaluación CSC y modelo KMAT, entregado a los directores o personal responsable de las escuelas en las reuniones de órgano colegiado de cada zona escolar del estado de Guanajuato. Conformado por un total de 62 ítems, de los cuales 12 son preguntas respecto al perfil del encuestado y 50 ítems corresponden a los factores analizados en esta investigación (ver tabla 2), que incluyen tres preguntas abiertas, cuatro ítems cerrados: tres cuestionamientos de los principales procesos que describen a la gestión del conocimiento y un ítem de los factores que lo obstaculizan, las preguntas o ítems restantes utilizan escala Likert en tres o siete categorías de respuesta. Los ítems son agrupados en siete dimensiones (ver tabla 2). 
Tabla 2. Instrumento de Gestión del Conocimiento en Instituciones de Educación Básica

\begin{tabular}{|c|c|c|c|}
\hline Núm. & Ítem & Núm. & Ítem \\
\hline 13 & Entiende que es conocimiento & 38 & $\begin{array}{l}\text { Requiero sistemas computacionales } \\
\text { para gestionar el conocimiento }\end{array}$ \\
\hline 14 & Importancia del conocimiento & 39 & $\begin{array}{l}\text { Requiero tecnologías de la información } \\
\text { y la comunicación }\end{array}$ \\
\hline 15 & Que es gestión del conocimiento & 40 & Requiero benchmarking \\
\hline 16 & Cómo gestionar el conocimiento & 41 & $\begin{array}{l}\text { Requiero sistemas de apoyo en } \\
\text { procesos operativos y administrativos }\end{array}$ \\
\hline 17 & $\begin{array}{l}\text { Como transferir conocimiento tácito a } \\
\text { explicito }\end{array}$ & 42 & Motivos para tener procesos de GC \\
\hline 18 & $\begin{array}{l}\text { Como identificar y difundir mejores } \\
\text { practicas }\end{array}$ & 43 & Cinco procesos mas importantes \\
\hline 19 & $\begin{array}{l}\text { Conozco sistemas de aprendizajes auto } \\
\text { gestionados }\end{array}$ & 44 & $\begin{array}{l}\text { Procesos ya implantados o en } \\
\text { implantación }\end{array}$ \\
\hline 20 & $\begin{array}{l}\text { Conozco sistemas computacionales para } \\
\text { gestionar el conocimiento }\end{array}$ & 45 & $\begin{array}{l}\text { Año de inicio del primer proceso de } \\
\text { GC implantado }\end{array}$ \\
\hline 21 & $\begin{array}{l}\text { Conozco sistemas computacionales de } \\
\text { aprendizaje distribuido }\end{array}$ & 46 & Contaron con apoyo de un líder \\
\hline 22 & $\begin{array}{l}\text { Conozco la creación de comunidades } \\
\text { aprendizaje }\end{array}$ & 47 & Contaron con recursos financieros \\
\hline 23 & $\begin{array}{l}\text { Conozco sistemas de aprendizaje en } \\
\text { competencias }\end{array}$ & 48 & Es o era una iniciativa estratégica \\
\hline 24 & $\begin{array}{l}\text { Entiendo como la gestión del conocimiento } \\
\text { favorece la competitividad }\end{array}$ & 49 & Tienen ímpetu y apoyo \\
\hline 25 & $\begin{array}{l}\text { Mi institución requiere gestionar el } \\
\text { conocimiento }\end{array}$ & 50 & Contaron con consultores externos \\
\hline 26 & $\begin{array}{l}\text { Personas en mi institución hablan de } \\
\text { gestión del conocimiento }\end{array}$ & 51 & $\begin{array}{l}\text { Fueron implantados en momento } \\
\text { adecuado }\end{array}$ \\
\hline 27 & $\begin{array}{l}\text { La gestión del conocimiento esta dentro de } \\
\text { la agenda estratégica }\end{array}$ & 52 & $\begin{array}{l}\text { Tecnologìas que se utilizan para } \\
\text { implantar las inicitivas de GC }\end{array}$ \\
\hline 28 & $\begin{array}{l}\text { Yo implantaría gestión del conocimiento } \\
\text { en mi escuela }\end{array}$ & 53 & Área responsable de la implantación \\
\hline 29 & $\begin{array}{l}\text { Requiero entender gestión del } \\
\text { conocimiento }\end{array}$ & 54 & Principales obstáculos \\
\hline 30 & Requiero fundamentarlo línea estratégica & 55 & Se obtuvieron los resultados esperados \\
\hline 31 & Requiero capacitación & 56 & Beneficios obtenidos \\
\hline 32 & $\begin{array}{l}\text { Requiero identificar conocimiento } \\
\text { organizacional relevante }\end{array}$ & 57 & Iniciar gestión del conocimiento \\
\hline 33 & $\begin{array}{l}\text { Requiero metodologías que guíen el } \\
\text { proceso }\end{array}$ & 58 & Sistemas más útiles \\
\hline 34 & Requiero casos de aplicación & 59 & $\begin{array}{l}\text { Cuentan con un puesto de alto nivel } \\
\text { para la gestión del conocimiento }\end{array}$ \\
\hline 35 & Requiero consultores expertos & 60 & $\begin{array}{l}\text { Prioridad de gestión del conocimiento } \\
\text { para las organizaciones de clase } \\
\text { mundial }\end{array}$ \\
\hline 36 & Requiero bibliografía & 61 & $\begin{array}{l}\text { Prioridad de gestión del conocimiento } \\
\text { para las escuelas de prestigio }\end{array}$ \\
\hline
\end{tabular}

Revista de Gestão e Secretariado-GeSec, São Paulo, v. 8, n. 2, p 20-39, Mai./Ago. 2017. 
37 Requiero contacto con instituciones con gestión del conocimiento

62 Participaría en grupo o asociación especializada en gestión del conocimiento

Fuente: Elaboración propia.

\subsection{Datos técnicos de la investigación}

En la tabla 3 se observan los datos técnicos de la investigación de la gestión del conocimiento de las Instituciones de Educación Básica del estado de Guanajuato, México.

\section{Tabla 3. Datos técnicos de la investigación}

\begin{tabular}{|l|l|}
\hline Universo & $\begin{array}{l}508 \text { docentes y directivos de14 Instituciones de Educación Básica públicas } \\
\text { y privadas de la zona escolar 557 Primaria y 27 de la zona escolar 506 } \\
\text { Secundaria, del estado de Guanajuato, México. (Fuente: SEG 2013). }\end{array}$ \\
$\begin{array}{l}\text { País de origen } \\
\text { Muestra probabilística } \\
\text { estratificada }\end{array}$ & México \\
Error muestral & 219, con una muestra final de 222 encuestas \\
Nivel de confianza & $5 \%$ \\
Método de muestreo & $95 \%$ \\
Recolección de datos & $\begin{array}{l}\text { Aleatorio simple } \\
\text { Cuestionario entregado a directores o personal responsable de las escuelas } \\
\text { en las reuniones de órgano colegiado de cada zona escolar para que fuera } \\
\text { aplicado a su plantilla docente y personal directivo. }\end{array}$ \\
& $\begin{array}{l}\text { Análisis Factorial Exploratorio, con factores de extracción de componentes } \\
\text { principales, utilizando el método Varimax de rotación ortogonal. }\end{array}$ \\
Técnicas estadísticas & SPSS versión 21.0 \\
Programas estadísticos &
\end{tabular}

Fuente: Elaboración propia.

\subsection{Matriz metodológica}

La matriz metodológica define el modelo propuesto en función de la relación que existe entre las variables propuestas por el modelo KMAT y las dimensiones del CSC con la Gestión del conocimiento en las Instituciones de Educación a Nivel Básico. Evaluando su consistencia de contenido, el instrumento se sometió a expertos eliminando los ítems 42, 45 y 52, por ser cuestionamientos abiertos, realizando las adecuaciones pertinentes. En la tabla 4 se muestran las variables y dimensiones de este estudio, así como los 47 ítems que se utilizan del instrumento original. 
Tabla 4. Matriz Metodológica

\section{Variable: Liderazgo Código: L}

\begin{tabular}{|c|c|c|c|}
\hline Dimensión & Código & Indicador & Ítem \\
\hline Entendimiento & $\mathrm{E}$ & $\begin{array}{l}\text { Definir conocimiento, su importancia, definir gestión del } \\
\text { conocimiento, como se gestiona y aplica. }\end{array}$ & $\begin{array}{l}13-19 \\
22-24\end{array}$ \\
\hline Intención & I & $\begin{array}{l}\text { Necesidad de implementar, incluirlo en la agenda } \\
\text { estratégica y tomar la decisión de su implementación. }\end{array}$ & $25-28$ \\
\hline Requerimientos & $\mathrm{R}$ & Entender como se gestiona el conocimiento & $\begin{array}{l}29-37 \\
40-41\end{array}$ \\
\hline Práctica & $\mathrm{P}$ & $\begin{array}{l}\text { Qué motiva la gestión del conocimiento, el apoyo recibido, } \\
\text { los recursos asignados, área responsable y obstáculos para } \\
\text { la implementación. }\end{array}$ & $\begin{array}{l}43-44 \\
46-51 \\
53-54\end{array}$ \\
\hline Resultados & RS & El logro de los resultados & $55-56$ \\
\hline Visión futura & VF & $\begin{array}{l}\text { Visión de implementar gestión del conocimiento dentro de } \\
\text { su institución y su prioridad para las organizaciones. }\end{array}$ & $57-62$ \\
\hline
\end{tabular}

Variable: Cultura Organizacional Código: CO

\begin{tabular}{|l|c|l|l|}
\hline Dimensión & Código & Indicador & Ítem \\
\hline Entendimiento & $\mathrm{E}$ & $\begin{array}{l}\text { Definir conocimiento, su importancia, definir gestión del } \\
\text { conocimiento, como se gestiona y aplica dentro de la } \\
\text { institución. }\end{array}$ & $\begin{array}{l}13-19 \\
22-24\end{array}$ \\
\hline Requerimientos & $\mathrm{R}$ & Identificación de la base del conocimiento organizacional. & 29 \\
& & & $31-37$ \\
& $\mathrm{P}$ & Iniciativa estratégica, motivación, apoyo y obstáculos. & $40-41$ \\
\hline Práctica & & & $48-44$ \\
& $\mathrm{RS}$ & Logro de los resultados & 54 \\
\hline Resultados & $\mathrm{VF}$ & $\begin{array}{l}\text { Prioridad de las organizaciones por la gestión del } \\
\text { conocimiento }\end{array}$ & $55-56$ \\
\hline Visión futura & & 58 y 60- \\
& & 61
\end{tabular}

Variable: Tecnología de la Información y la Comunicación Código: TIC

\begin{tabular}{|l|c|l|l|}
\hline \multicolumn{1}{|c|}{ Dimensión } & Código & \multicolumn{1}{c|}{ Indicador } & \multicolumn{1}{c|}{ Ítem } \\
\hline Entendimiento & $\mathrm{E}$ & Sistemas computacionales para gestión del conocimiento & $20-21$ \\
\hline Requerimientos & $\mathrm{R}$ & Sistemas computacionales para gestión del conocimiento & $38-39$ \\
\hline Práctica & $\mathrm{P}$ & Tecnologías utilizadas & $43-44$, \\
& & & $46-48$ \\
\hline Visión futura & VF & Sistemas más útiles & 58 \\
\hline & &
\end{tabular}

\begin{tabular}{|l|c|l|l|}
\hline \multicolumn{1}{|c|}{ Dimensión } & Código & \multicolumn{1}{c|}{ Variable: Medición Código: M } & \multicolumn{1}{c|}{ Indicador } \\
\hline Entendimiento & E & $\begin{array}{l}\text { De tácito a explicito, mejores prácticas, aprendizaje auto- } \\
\text { gestionado, comunidades de práctica }\end{array}$ & $\begin{array}{l}17-19 \\
22-23\end{array}$ \\
\hline Requerimientos & R & Identificación del conocimiento organizacional interno. & $29,32-33$ \\
\hline Práctica & P & Procesos más importantes & $43-44$ \\
\hline Resultados & RS & Resultados logrados & $55-56$ \\
\hline Visión futura & VF & Sistemas más útiles & 58 \\
\hline
\end{tabular}

Fuente: Elaboración propia a partir del modelo KMAT (Andersen (1999) y la evaluación de factores del CSC (2001).

Revista de Gestão e Secretariado-GeSec, São Paulo, v. 8, n. 2, p 20-39, Mai./Ago. 2017. 
En base al modelo KMAT y a la evaluación de factores del CSC se plantean las siguientes hipótesis:

$\mathrm{H}_{1}$. La gestión del conocimiento de las Instituciones de Educación Básica, está determinado por el entendimiento, intención, requerimientos, práctica, resultados y visión futura.

$\mathrm{H}_{2}$. La gestión del conocimiento de las Instituciones de Educación Básica, está determinado por el Liderazgo, Cultura organizacional, Tecnologías de la información y comunicación y medición.

\section{Análisis y discusión de resultados}

\subsection{Validez inicial del Instrumento}

Procediendo a realizar el análisis de esta investigación, primeramente se obtuvo la consistencia interna de las dimensiones del estudio sobre el instrumento original, mostrando en la tabla 5, que la dimensión intención es no aceptable con un alfa de Cronbach $\alpha=0.484$, así mismo la dimensión de visión futura presenta un valor negativo de $\alpha=-0.077$ por inconsistencia en la covarianza negativa resultante de la escala utilizada (de acuerdo con el criterio de George y Mallery, 2003), por lo que se procedió a realizar el análisis factorial, a fin de obtener una escala robusta.

Tabla 5. Confiabilidad de la escala original por dimensión de la evaluación CSC

\begin{tabular}{|lccc|}
\hline \multicolumn{1}{|c}{ Dimensión } & $\begin{array}{c}\text { Ítems } \\
\text { propuestos CSC }\end{array}$ & $\begin{array}{c}\text { Ítems } \\
\text { validos }\end{array}$ & $\begin{array}{c}\text { Alfa de } \\
\text { Cronbach }\end{array}$ \\
\hline Entendimiento & 12 & 12 & 0.873 \\
Intención & 4 & 4 & 0.484 \\
Requerimientos & 13 & 13 & 0.860 \\
Práctica & 13 & 10 & 0.920 \\
Resultados & 2 & 2 & 0.602 \\
Visión futura & 6 & 6 & -0.077 \\
Instrumento & $\mathbf{5 0}$ & $\mathbf{4 7}$ & $\mathbf{0 . 8 4 0}$ \\
\hline
\end{tabular}

Fuente: Elaboración propia.

\subsection{Análisis factorial}

Realizando el análisis por el método de extracción de análisis de componentes principales, en la tabla 6 , se presenta la matriz de varianza total explicada y el porcentaje de la varianza que representa para cada uno de los componentes, determinando que son 12 componentes principales donde se concentra el $70.289 \%$ de la varianza acumulada de la escala original. 
Tabla 6. Varianza total explicada

\begin{tabular}{|c|c|c|c|c|c|c|c|c|c|}
\hline \multirow[t]{2}{*}{ Componente } & \multicolumn{3}{|c|}{ Auto valores iniciales } & \multicolumn{3}{|c|}{$\begin{array}{l}\text { Sumas de las saturaciones al } \\
\text { cuadrado de la extracción }\end{array}$} & \multicolumn{3}{|c|}{$\begin{array}{l}\text { Suma de las saturaciones al } \\
\text { cuadrado de la rotación }\end{array}$} \\
\hline & Total & $\begin{array}{c}\% \text { de la } \\
\text { varianza }\end{array}$ & $\%$ acumulado & Total & $\begin{array}{c}\% \text { de la } \\
\text { varianza }\end{array}$ & $\%$ acumulado & Total & $\begin{array}{c}\% \text { de la } \\
\text { varianza }\end{array}$ & $\%$ acumulado \\
\hline 1 & 8,195 & 17,436 & 17,436 & 8,195 & 17,436 & 17,436 & 8,137 & 17,313 & 17,313 \\
\hline 2 & 6,689 & 14,231 & 31,667 & 6,689 & 14,231 & 31,667 & 5,539 & 11,785 & 29,098 \\
\hline 3 & 5,355 & 11,394 & 43,061 & 5,355 & 11,394 & 43,061 & 4,626 & 9,842 & 38,940 \\
\hline 4 & 1,998 & 4,251 & 47,312 & 1,998 & 4,251 & 47,312 & 2,737 & 5,824 & 44,764 \\
\hline 5 & 1,842 & 3,919 & 51,231 & 1,842 & 3,919 & 51,231 & 1,960 & 4,171 & 48,935 \\
\hline 6 & 1,570 & 3,341 & 54,572 & 1,570 & 3,341 & 54,572 & 1,714 & 3,648 & 52,582 \\
\hline 7 & 1,370 & 2,915 & 57,487 & 1,370 & 2,915 & 57,487 & 1,527 & 3,249 & 55,832 \\
\hline 8 & 1,307 & 2,780 & 60,267 & 1,307 & 2,780 & 60,267 & 1,437 & 3,058 & 58,889 \\
\hline 9 & 1,288 & 2,739 & 63,007 & 1,288 & 2,739 & 63,007 & 1,420 & 3,020 & 61,910 \\
\hline 10 & 1,268 & 2,699 & 65,706 & 1,268 & 2,699 & 65,706 & 1,353 & 2,878 & 64,788 \\
\hline 11 & 1,107 & 2,355 & 68,061 & 1,107 & 2,355 & 68,061 & 1,332 & 2,834 & 67,622 \\
\hline 12 & 1,047 & 2,228 & 70,289 & 1,047 & 2,228 & 70,289 & 1,253 & 2,666 & 70,289 \\
\hline 13 & ,988 & 2,101 & 72,390 & & & & & & \\
\hline 14 & ,921 & 1,960 & 74,349 & & & & & & \\
\hline 15 & ,852 & 1,812 & 76,161 & & & & & & \\
\hline 16 & ,789 & 1,678 & 77,839 & & & & & & \\
\hline 17 & ,718 & 1,528 & 79,368 & & & & & & \\
\hline 18 & ,703 & 1,496 & 80,863 & & & & & & \\
\hline 19 & ,689 & 1,467 & 82,330 & & & & & & \\
\hline 20 & ,632 & 1,345 & 83,675 & & & & & & \\
\hline 21 & ,628 & 1,336 & 85,011 & & & & & & \\
\hline 22 &, 567 & 1,207 & 86,218 & & & & & & \\
\hline 23 & ,543 & 1,155 & 87,374 & & & & & & \\
\hline 24 & ,504 & 1,073 & 88,446 & & & & & & \\
\hline 25 & ,474 & 1,008 & 89,455 & & & & & & \\
\hline 26 & ,460 & ,978 & 90,433 & & & & & & \\
\hline 27 & ,409 & ,870 & 91,303 & & & & & & \\
\hline 28 & ,403 & ,857 & 92,160 & & & & & & \\
\hline 29 & ,370 & ,787 & 92,947 & & & & & & \\
\hline 30 & ,360 & ,767 & 93,714 & & & & & & \\
\hline 31 &, 326 & ,693 & 94,407 & & & & & & \\
\hline 32 & ,299 & ,636 & 95,043 & & & & & & \\
\hline 33 & ,270 & ,575 & 95,618 & & & & & & \\
\hline 34 & ,247 & ,525 & 96,143 & & & & & & \\
\hline 35 & ,233 & ,495 & 96,638 & & & & & & \\
\hline 36 & ,206 & ,438 & 97,076 & & & & & & \\
\hline 37 & ,194 & ,412 & 97,488 & & & & & & \\
\hline 38 & ,179 & ,381 & 97,870 & & & & & & \\
\hline 39 & ,168 & ,357 & 98,226 & & & & & & \\
\hline 40 & , 155 & ,330 & 98,557 & & & & & & \\
\hline 41 & ,145 & ,308 & 98,865 & & & & & & \\
\hline 42 & ,118 & ,252 & 99,117 & & & & & & \\
\hline 43 & ,115 & ,244 & 99,361 & & & & & & \\
\hline 44 & ,089 & ,189 & 99,550 & & & & & & \\
\hline 45 & ,082 & , 175 & 99,726 & & & & & & \\
\hline 46 & ,077 & ,164 & 99,890 & & & & & & \\
\hline 47 & ,052 & ,110 & 100,000 & & & & & & \\
\hline
\end{tabular}

Método de extracción: Análisis de Componentes principales.

Revista de Gestão e Secretariado-GeSec, São Paulo, v. 8, n. 2, p 20-39, Mai./Ago. 2017. 
Considerando valores absolutos de carga factorial mínimo de 0.450, la matriz de componentes descrita en la tabla 7, permite observar que los factores 25 y 27 tienen un valor absoluto menor a 0.450 , por lo cual se eliminan. Así mismo se eliminaron los ítems 26, 28, 57, 58,59 y 62, cuyos factores caen de manera individual en los componentes 7,8,9,10, 11 y 12. Con una medida de adecuación muestral de KMO corresponde a 0.797.

\section{Tabla 7. Matriz de componentes rotados}

\begin{tabular}{|c|c|c|c|c|c|c|c|c|c|c|c|c|}
\hline \multirow[t]{2}{*}{ Ítem } & \multicolumn{12}{|c|}{ Componente } \\
\hline & 1 & 2 & 3 & 4 & 5 & 6 & 7 & 8 & 9 & 10 & 11 & 12 \\
\hline Entiende que es conocimiento & &, 757 & & & & & & & & & & \\
\hline Importancia del conocimiento & &, 768 & & & & & & & & & & \\
\hline Que es gestión del conocimiento & & 795 & & & & & & & & & & \\
\hline Cómo gestionar el conocimiento & & 804 & & & & & & & & & & \\
\hline Como transferir conocimiento tácito a explicito & & 765 & & & & & & & & & & \\
\hline Como identificar y difundir mejores practicas & & 661 & & & & & & & & & & \\
\hline Conozco sistemas de aprendizajes auto gestionados & &, 544 & & & & & & & & & & \\
\hline $\begin{array}{l}\text { Conozco sistemas computacionales para gestionar el } \\
\text { conocimiento }\end{array}$ & & & & 851 & & & & & & & & \\
\hline Conozco sistemas computacionales de aprendizaje distribuido & & & & 765 & & & & & & & & \\
\hline Conozco la creación de comunidades aprendizaje & & & &, 729 & & & & & & & & \\
\hline Conozco sistemas de aprendizaje en competencias & & 646 & & & & & & & & & & \\
\hline $\begin{array}{l}\text { Entiendo como la gestión del conocimiento favorece la } \\
\text { competitividad }\end{array}$ & & ,728 & & & & & & & & & & \\
\hline Mi institución requiere gestionar el conocimiento & & & & & & & & & & & & \\
\hline Personas en mi institución hablan de gestión del conocimiento & & & & & & & & & ,543 & & & \\
\hline $\begin{array}{l}\text { La gestión del conocimiento esta dentro de la agenda } \\
\text { estratégica }\end{array}$ & & & & & & & & & & & & \\
\hline Yo implantaría gestión del conocimiento en mi escuela & & & & & & &, 780 & & & & & \\
\hline Requiero entender gestión del conocimiento & & &, 528 & & & & & & & & & \\
\hline Requiero fundamentarlo línea estratégica & & & 711 & & & & & & & & & \\
\hline Requiero capacitación & & & 590 & & & & & & & & & \\
\hline Requiero identificar conocimiento organizacional relevante & & & 710 & & & & & & & & & \\
\hline Requiero metodologías que guíen & & & 612 & & & & & & & & & \\
\hline Requiero casos de aplicación & & & 668 & & & & & & & & & \\
\hline Requiero consultores expertos & & & 659 & & & & & & & & & \\
\hline Requiero bibliografía & & & 636 & & & & & & & & & \\
\hline $\begin{array}{l}\text { Requiero contacto con instituciones con gestión del } \\
\text { conocimiento }\end{array}$ & & &, 596 & & & & & & & & & \\
\hline $\begin{array}{l}\text { Requiero sistemas computacionales para gestionar el } \\
\text { conocimiento }\end{array}$ & & & & & ,726 & & & & & & & \\
\hline Requiero tecnologías de la información y la comunicación & & & & & 808 & & & & & & & \\
\hline Requiero benchmarking & & &, 522 & & & & & & & & & \\
\hline $\begin{array}{l}\text { Requiero sistemas de apoyo en procesos operativos y } \\
\text { administrativos }\end{array}$ & & & 464 & & & & & & & & & \\
\hline Cinco procesos mas importantes & 659 & & & & & & & & & & & \\
\hline Procesos ya implantados o en implantación & 510 & & & & & & & & & & & \\
\hline Contaron con apoyo de un líder & 847 & & & & & & & & & & & \\
\hline Contaron con recursos financieros & 866 & & & & & & & & & & & \\
\hline Es o era una iniciativa estratégica & 859 & & & & & & & & & & & \\
\hline Tienen ímpetu y apoyo & 890 & & & & & & & & & & & \\
\hline Contaron con consultores externos &, 888 & & & & & & & & & & & \\
\hline Fueron implantados en momento adecuado &, 861 & & & & & & & & & & & \\
\hline Área responsable de la implantación &, 853 & & & & & & & & & & & \\
\hline
\end{tabular}

Revista de Gestão e Secretariado-GeSec, São Paulo, v. 8, n. 2, p 20-39, Mai./Ago. 2017. 


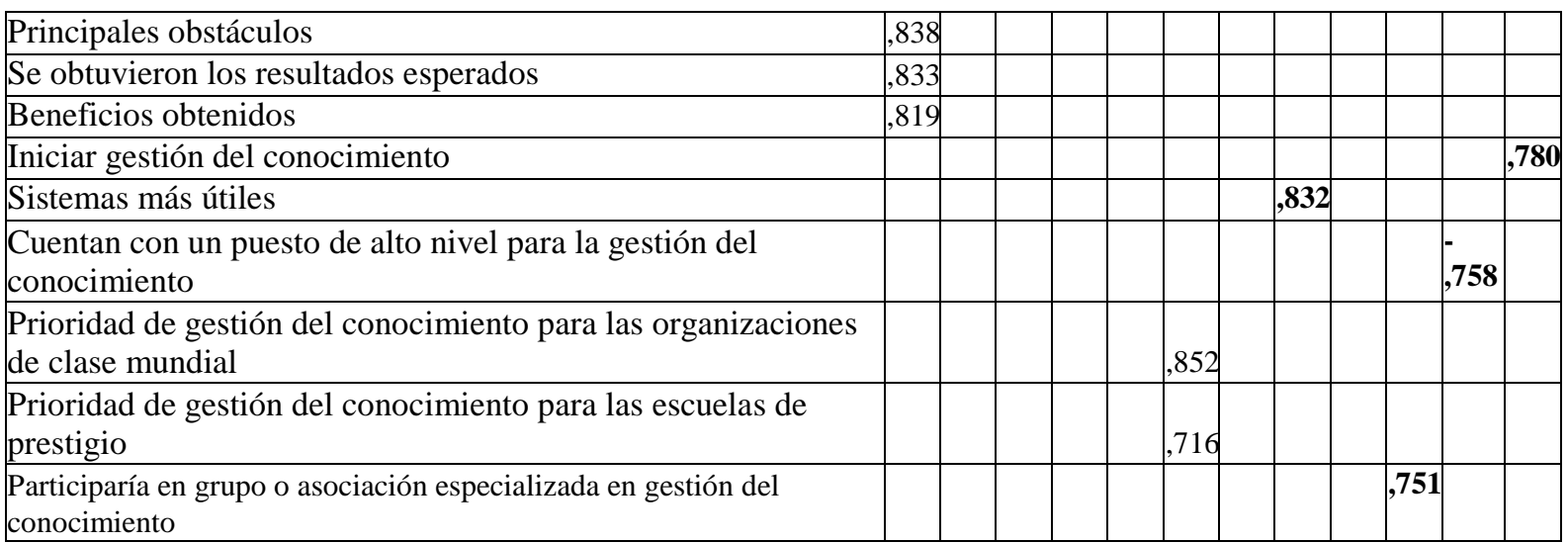

Método de extracción: Análisis de componentes principales.

Método de rotación: Normalización Varimax con Kaiser. a

A la rotación ha convergido en 14 iteraciones.

Eliminando los 8 factores de la dimensión intención $(25,26,27,28)$ y visión futura $(57$, $58,59,62)$, se aplico el AFE a los 39 ítems, obteniendo una varianza total explicada de $77.241 \%$, con una medida de adecuación muestral con un coeficiente KMO a 0.824 , considerado un valor alto (Kaiser 1970, 1974). Contrastando la hipótesis nula con la prueba de esfericidad de Bartlet (1950), se encontró una correlación significativa de 0.000, por tanto el modelo factorial es adecuado para explicar los datos.

\subsection{Validez final del Instrumento}

En relación a las dimensiones del estudio, la validez del instrumento final queda con cinco de las seis dimensiones propuestas, quedando sin validar la dimensión de intención. Con una confiabilidad en el rango de bueno en el análisis de las dimensiones de entendimiento y requerimientos; en el rango de excelente en práctica; mientras que visión futura y resultados el rango de cuestionable, aunque de acuerdo con Nunnally (1978) en las primeras fases de la investigación valores de 0.6 son suficiente (ver tabla 8).

Tabla 8. Confiabilidad de Ítems por dimensión e instrumento final

\begin{tabular}{|l|cc|}
\hline \multicolumn{1}{|c|}{ Dimensión } & $\begin{array}{c}\text { Ítems } \\
\text { validos }\end{array}$ & Alfa de Cronbach \\
\hline Entendimiento & 12 & 0.873 \\
Intención & - & - \\
Requerimientos & 13 & 0.860 \\
Práctica & 10 & 0.920 \\
Resultados & 2 & 0.602 \\
Visión futura & 2 & 0.657 \\
Instrumento & $\mathbf{3 9}$ & $\mathbf{0 . 8 6 8}$ \\
\hline
\end{tabular}

Fuente: Elaboración propia.

Pese a que los 39 ítems resultantes del instrumento tienen un valor de $\alpha=0.868$, este coeficiente cuando es usado para evaluar una prueba que explora dos o más dimensiones o variables distintas aún cuando formen parte de un mismo constructo, de acuerdo con Oviedo y Campos (2005), se corre el riesgo de subestimar la consistencia interna, proponiendo que lo más indicado es calcular el valor de alfa de Cronbach para cada grupo de ítems que componen

Revista de Gestão e Secretariado-GeSec, São Paulo, v. 8, n. 2, p 20-39, Mai./Ago. 2017. 
cada variable. Por lo que en la tabla 9, se presentan los resultados obtenidos en la determinación de la confiabilidad de los ítems agrupados por las variables del modelo KMAT, observando que la variables medición tiene un valor bajo en su confiabilidad $\alpha=0.523$. Aunque en las primeras fases de la investigación se puede considerar suficiente un Alfa de Cronbach de 0.5 a 0.6 (Nunnally, 1978). Cabe aclarar que cada variable del modelo KMAT contempla una serie de ítems en las que incluyen factores de la evaluación CSC (ver tabla 4).

Tabla 9. Confiabilidad de Ítems por variable del modelo KMAT

\begin{tabular}{|l|c|c|}
\hline \multicolumn{1}{|c|}{ Variable } & $\begin{array}{c}\text { Ítems } \\
\text { validos }\end{array}$ & $\begin{array}{c}\text { Alfa de } \\
\text { Cronbach }\end{array}$ \\
\hline Liderazgo & 35 & 0.866 \\
\hline Cultura & 30 & 0.811 \\
\hline Tecnología & 9 & 0.745 \\
\hline Medición & 12 & 0.523 \\
\hline
\end{tabular}

Fuente: Elaboración propia.

\section{CONSIDERACIONES FINALES}

El objetivo de este trabajo de investigación fue obtener un instrumento robusto que evaluará la Gestión del conocimiento en Instituciones de Educación Básica en el Estado de Guanajuato, México, a partir de la adaptación del modelo de diagn stico "Knowledge Management Assessment Tool” y la evaluación CSC. El estudio permitió determinar un instrumento con un total de 39 ítems.

En relación a las hipótesis planteadas, se acepta parcialmente la hip tesis " $\mathrm{H}_{1}$. La gestión del conocimiento de las Instituciones de Educación Básica, está determinado por el entendimiento, intención, requerimientos, práctica, resultados y visión futura", porque se elimina la dimensión intención. Aceptando la hipótesis $\mathrm{H}_{2}$. "La gesti n del conocimiento de las Instituciones de Educación Básica, está determinado por el Liderazgo, Cultura organizacional, Tecnologías de la informaci n y comunicaci n y medici n", porque aunque el factor medición tiene un valor de confiabilidad bajo, de manera general se obtuvo un instrumento robusto.

La limitante de esta investigación es que se enfoca solo a una región de México, por lo que se sugiere ampliar el estudio a toda la republica mexicana. Otra limitante es la baja confiabilidad de la variable medición, por lo que se recomienda realizar un análisis confirmatorio y ampliar el número de cuestionamientos en esta variable a fin de fortalecer los resultados.

La principal aportación de este estudio es que se involucran elementos del conocimiento, resultados obtenidos y visión a largo plazo con las dimensiones entendimiento, intención, requerimientos, práctica, resultados y visión futura, con los factores que motivan la gestión de conocimiento como son el liderazgo cultura organizacional, tecnologías de la información y comunicación y medición, para obtener una escala robusta de Gestión del conocimiento en Instituciones de Educación Básica. 


\section{REFERENCIAS}

Aguerrondo, I. (1999). El nuevo paradigma de la educación para el siglo. Recuperado de http://www.oei.es/administracion/aguerrondo.htm

Alwis, R.S.D. y Hartmann, E. (2008). The use of tacit knowledge within innovative companies: knowledge management in innovative enterprises. Journal of Knowledge Management, 12 (1), 133-147.

Andersen, A. (1999). KMAT. The Knowledge Management Assessment Tool. London: Arthur Andersen.

APQC. (2015). 2015 Knowledege Management Priorities. Data Report. APQC.

Ayustre, A. Gros B y Valdivielso, S. (2012). Sociedad del Conocimiento. Perspectiva Pedagogica. XXXI Seminario Ineruiniverisatiro de Teoria de la Educación. Plascencia. España. Bednar, A., Cunningham, D., Duffy, T. y Perry, J. (1992). Theory into practice: How do we link? . En T. Duffy y D. Jonassen (Eds.), Constructivism and the technology of instruction: a conversation (pp. 17-34). Hillsdale, NJ, EE.UU: Lawrence Erlbaum Associates.

Canton, I. (2009). Narraciones de la escuela. Barcelona, España: Davinci Continental.

Casas, R. (2001). Problemas en la producción y la transferencia del conocimiento. Tesis de maestría en ciencia, tecnologia y sociedad no publicada, Universidad Nacional de Quilmes, Bernal, Argentina.

Castro, C. (2011). Gestión del conocimiento en una organización intensiva en conocimiento: el caso de un centro de investigación de excelentcia en Colombia. Universidad del Valle. Civilizar , 52-70.

Dutta, S. y De Meyer, A. (1997). Knowledge Management at Arthur Andersen (Denmark): Building Assets in Real Time and in Virtual Space. INSEAD. Fontainebleau

George, D., y Mallery, P. (2003). SPSS for Windows step by step: A simple guide and reference. 11.0 update (4th ed.). Boston: Allyn y Bacon.

Chungchi, H. y Chie-Chug, L. (2013). Knowledge management development in an elementary school-Fongsiang as a case in Taiwan. Journal of education and review, 1 (5), 33-42

CIC-IADE. (2003). El modelo intellectus: medición y gestión del capital intelectual. Institutto Universitario de Administración y Dirección de Empresas. Madrid: Documento Intellectus No.5.

CINE 2011. (2013). Clasificación Internacional Normalizada de la Educación. Instituto de Estadistíca. Montreal, Québec: UNESCO.

Revista de Gestão e Secretariado-GeSec, São Paulo, v. 8, n. 2, p 20-39, Mai./Ago. 2017. 
CSC (2001). Administración del conocimiento en México: entendimiento, intención, práctica, resultados y visión a futuro. (Informe). Centro de Sistemas de Conocimiento, Tecnológico de Monterrey, Monterrey, NL, México.

Cranfield, D., y Taylor, J. (2008). Knowledege management and higher education: A Uk case study. The electronic journal of knowledge management , II (6), 85-100.

Dalman, C y Aubert, J (2001). China and the knowledge economy. Siezing the 21st Century, WBI Development Studies, World Bank Institute y World Bank, East Asia and Pacific Region, Washington D.C.

Davenport, T. y Prusak, L. (2000). Working knowledge: how organizations manage what they know (2nd ed.). Boston, MA, EE.UU: Harvard Business Review. Press.

Delors, J. (1996). La educación encierra un tesoro. Informe a la UNESCO de la Comisión Internacional sobre la educación para el siglo XXI. Madrid, España: Santillana ediciones UNESCO.

Díaz Barriga, F. y Lugo, E. (2003). Desarrollo del currículo e innovación. En A. Díaz Barriga, La investigación curricular en México. La década de los noventa (pp. 63-123). México: COMIE-CESU-SEP.

DOF. (2013). Programa sectorial de educación 2013-2018. Secretaria de Educación Pública. México: Diario oficial de la federación.

Gairín, J., y Rodríguez-Gómez, D. (2012). El modelo Accelera de creación y gestión del conocimiento en el ámbito educativo. Revista de educación , 357, 633-646.

García, I., Camarena, C., y Gil, M. (2009). Descripción de una herramienta tecnológica para gestionar el capital intelectual del Instituto Tecnológico de Sonora. Retrieved 12 de Octubre de 2012 from Instituto Tecnológico de Sonora: http:antiguo.itson.mx/publicaciones/contaduría/CIN2009/10.pdf

Gianini, V.C., y Gerardin, J. U. (2010). Gestão Educacional: A Atuação do Profissional Secretário nas Organizações Educacionais. Revista de Gestão e Secretariado, 1(2), 32-52. http://dx.doi.org/10.7769/gesec.v1i2.32

Hargreaves, A. (2003). Enseñar en la sociedad del conocimiento. Barcelona: Ocatedro. Hawryszkiewycz, I. (2009). Knowledge management. Organizing knowlede based enterprises. Sydney, Australia: Palgrave Macmillan.

Hernández, E. y Velasco, A. (1990). Productividad y competitividad de las manufacturas mexicanas, 1960.1985. Comercio Exterior , 40 (2), 658-666.

Jaime, A.; Gardoni, M.; Mosca, J.; Vinck, D. (2005). BASIC Lab: a software tool for supporting the production of knowledge in research organizations through the management of Revista de Gestão e Secretariado-GeSec, São Paulo, v. 8, n. 2, p 20-39, Mai./Ago. 2017. 
scientific concepts. Journal of Knowledge Management, 9(6), 53-66. http://dx.doi.org/10.1108/13673270510629963

Kline, P. (2000). Handbook of Pyschological testing. London: Routledge.

Lopera, E., y Quiroz, N. (2013). Caracterización de un modelo de gestión del conocimiento aplicable a las funciones universitarias de investigación y extensión:: Universidad del Rosario. Medellin Colombia: Tesis de maestria no publicada.

Martí, E. y Onrubia, J. (2002). Las teorías del aprendizaje escolar. Universidad Oberta. Catalunya, España:UOC/DIGITALIA.

Minakata, A. (2009). Gestión el conocimiento en educación y transformación de la escuela. Notas para un campo en construcción. Sinéctica 32.

Muñoz, A. (Abril-Junio de 2008). Escenarios e idnetidades del SNTE, ente el sistema educativo yel sistema político. RMIE, 13(37), 377-417.

Nissen, M. E. (2006). Harnessing knowledge dynamics: principled organizational knowing y learning. Hershey, PA, EE.UU: IRM Press.

Nonaka, I. y Takeuchi, H. (1995). La organización creadora del conocimiento. ¿Cómo las compañías japonesas crean la dinámica de la innovación? México DF, México: Oxford University Press.

Nunnally. (1978). Psychometric theory. New York: Mc Graw-Hill.

Organización para la Cooperación y el Desarrollo Económicos, OCDE. (1996). The Knowledge based economy. Recuperado de http://www.oecd.org/sti/sci-tech/1913021.pdf

Organización para la Cooperación y el Desarrollo Económicos, OCDE. (2000). Knowledge Management in the learning society. Recuperado de http://ocw.metu.edu.tr/file.php/118/Week11/oecd1.pdf

Organización para la Cooperación y el Desarrollo Económicos, OCDE. (2003). Medición de la gestión de conocimientos en las empresas: primeros resultados. Recuperado de www.oecd.org/edu/educationeconomyandsociety/31540414.pdf

Organización para la Cooperación y el Desarrollo Económicos, OCDE. (2012). Programa para la evaluación internacional de alumnos (PISA). PISA 2012 - Resultados México. Recuperado de http://www.oecd.org/pisa/keyfindings/PISA-2012-results-mexico-ESP.pdf

Organización para la Cooperación y el Desarrollo Económicos, OCDE. (2013). Education at a Gance 2013. Recuperado de http://dx.doi.org/10.1787/eag-2013-en

Organización para la Cooperación y el Desarrollo Económicos, OCDE. (2015). Education Policy Outlook 2015: Making reforms happen. Paris, Francia: OECD Publishing. doi:10 .1787/9789264225442-1-en 
Organización de Estados Iberoamericanos, OEI. (2010). 2021 Metas Educativas. La educación que queremos para la generación de los bicentenarios. Madrid, España: Organización de los Estados Iberoamericanos para la Educación, la Ciencia y la Cultura.

Oviedo, H., y Campo, A. (2005). Aproximación al uso del coeficiente alfa de Cronbach . Revista Colombiana de Psiquiatría, XXXIV (4), 572-580.

Peluffo, A y Catalán C (2002). Introducción a la gestión del conocimiento y su aplicación al sector público. Santiago de Chile: Instituto Latianoamericano del Caribe de Planificación Económica y Social.

Pérez, E.R., Medrano, L. (2010). Análisis Factorial Exploratorio: Bases conceptuales y metodológicas, Revista Argentina de Ciencias del Comportamiento 2(1), 58-66.

Ravela, P. (2000). La evaluación del desempeño docente para el desarrollo de las competencias profesionales. En E. Martín, \& F. Martinez Rizo, Avances y desafios en la evaluaicón educativa. Madrid, España: OEI.

Sallis, E. y Jones, G. (2002). Knowledge management in education: enhancing learning and education. Londres, Reino Unido: Kogan page limited.

Secretaria de Educación Pública (SEP) (2016). El Modelo Educativo 2016. México DF, México: Secretaria de Educación Pública.

Siemens, G. (2004). Conectivismo: Una teoría de aprendizaje para la era digital. Commons 2.5 Snelbecker, G. (1983). Learning theory, instructional theory, and psichoeducational designs. Nueva York, NY, EE.UU: Mac Graw-Hill.

Stankowsky, M. (2005). Advances in knowledge management: university research toward an academic discipline. En E. Butterworth-Heinemann, Creating the discipline of knowledge management; the latest in university research (pp.1-14). Amsterdam, Holanda. doi: 10.1016/B978-0-7506-7878-0.50005-3

Steward, T. (1997). La nueva riqueza de las organizaciones: capital intelectual. Buenos Aires, Argentina: Granica.

Taiwana, A. (2002). The knowledge managemente toolkit: practical technique for building a knowledege managemente system. New Yersy, Estados Unidos: Prentice Hall.

UNESCO. (2007). Compendio Mundial de la Educación 2007. Comparación de las estadísticas de educación en el mundo. Montreal: Instituto de Estadistica de la UNESCO.

Vigotsky, L. (1978). Interaction between learning and development. Mind and Society, 79-91.

Revista de Gestão e Secretariado-GeSec, São Paulo, v. 8, n. 2, p 20-39, Mai./Ago. 2017. 